\author{
B Stefanska \\ Department of Pharmacology and Therapeutics, McGill University, Montreal, Canada
}

\begin{abstract}
Correspondence
Barbara Stefanska, Department of Pharmacology and Therapeutics, McGill University, 3655 Sir William Osler Promenade \#1311, Montreal, Quebec, Canada H3G 1Y6. E-mail:

barbara.stefanska@mail.mcgill.ca
\end{abstract}

\section{Keywords}

curcumin; hepatic fibrosis; hepatic stellate cells; type 2 diabetes; RAGE

\section{Received}

1 March 2012

Revised

12 March 2012

Accepted

16 March 2012

A wide variety of beneficial effects have been attributed to curcumin, a major polyphenol from the golden spice Curcuma longa known as turmeric, including amelioration of severe complications of type 2 diabetes such as hepatic fibrosis, retinopathy, neuropathy and nephropathy. In the present issue of $B J P$, Lin and colleagues reveal new mechanisms by which curcumin inhibits the activation of hepatic stellate cells in vitro, a hallmark of non-alcoholic steatohepatitis and hepatic fibrogenesis associated with type 2 diabetes mellitus. They demonstrated that curcumin suppresses the advanced glycation end-products (AGEs)-mediated induction of the receptor for AGES (RAGE) gene expression by increasing PPAR $\gamma$ activity and stimulating de novo synthesis of glutathione. As a result, downstream elements of RAGE-activated pathways are inhibited, which prevents oxidative stress, inflammation and hepatic stellate cell activation. This report suggests that curcumin may have potential as an anti-fibrotic agent in type 2 diabetes and opens the door to the evaluation of curcumin therapeutic effects in liver conditions of different aetiology and in other disorders linked to the impairment of PPAR $\gamma$ activity, such as obesity and atherosclerosis.

\title{
LINKED ARTICLE
}

This article is a commentary on Lin et al., pp. 2212-2227 of this issue. To view this paper visit http://dx.doi.org/10.1111/ j.1476-5381.2012.01910.x

\section{Abbreviations}

AGEs, advanced glycation end-products; DNMTs, DNA methyltransferases; GSH, glutathione; HSCs, hepatic stellate cells; RAGE, receptors for AGEs; T2DM, type 2 diabetes mellitus

Curcumin (diferuloylmethane) is a bioactive food component present in the golden spice Curcuma longa known as turmeric that is an essential component of curry powder. Its chemopreventive and anti-cancer activities have been demonstrated in numerous studies and attributed to its antioxidant and anti-inflammatory properties (Basnet and SkalkoBasnet, 2011; Rogers et al., 2011). This polyphenol has been shown to reduce the risk of cancer (Basnet and Skalko-Basnet, 2011), heart disease (Siu, 2010), Alzheimer's disease (Belkacemi et al., 2011) and type 2 diabetes mellitus (T2DM) (Aggarwal, 2010). Curcumin supplementation improves diabetes-induced endothelial dysfunction by lowering plasma glucose levels, decreasing superoxide production and inhibiting protein kinase $\mathrm{C}$ in rats (Rungseesantivanon et al., 2010). By suppressing pro-inflammatory transcription factors and signal transducers and activating anti-inflammatory signalling pathways, curcumin reverses insulin resistance, hyperglycaemia, hyperlipidaemia and other symptoms linked to T2DM (Kang and Chen, 2009; Aggarwal, 2010; Tang and Chen, 2010). Ongoing clinical trials aim to show the effectiveness of curcumin in the reduction of atherosclerosis events in diabetic patients as well as in the prevention and delay of T2DM in patients with impaired glucose tolerance and insulin resistance.

T2DM results from resistance to insulin action and inadequate insulin secretion. The incidence of this disorder is significantly increasing worldwide. According to the World Health Organization (WHO), today 346 million people have diabetes and the number of diabetes-related deaths is estimated to almost double between 2005 and 2030 (WHO, 
Media Centre, 2011). Severe complications associated with diabetes, such as retinopathy, neuropathy, nephropathy, non-alcoholic steatohepatitis and hepatic fibrosis, have been the focus of research in this field in the last decade. Although effective ways of managing these complications are being developed, their molecular basis remains to be elucidated. One of the characteristic features of diabetes is the elevated level of blood glucose, hyperglycaemia, which is considered to be the principal cause of diabetic complications including hepatic fibrosis. The increased availability of glucose leads to the accelerated formation of advanced glycation endproducts (AGEs) that are non-enzymatic results of a chain of non-oxidative and oxidative reactions of sugars with proteins and/or lipids. AGEs interact with the receptor for AGEs (RAGE) located on the cell surface which subsequently stimulates different signalling pathways such as MAPK, JAK/STAT and PI3K, and produces pro-inflammatory responses with increased oxidative stress and cell growth (Ramasamy et al., 2009). AGEs-mediated increased proliferation of hepatic stellate cells (HSCs) was observed during hepatic fibrogenesis that was associated with RAGE up-regulation. Evidence has been obtained indicating the efficacy of curcumin at inhibiting the activation of HSCs in vitro and in vivo, which prevents hyperglycaemia- and hypercholesterolaemia-associated liver fibrosis (Kang et al., 2002; Kang and Chen, 2009; Tang and Chen, 2010). However, the precise mechanism through which curcumin exerts its hepatoprotective effect remains unclear.

In the present issue of the BJP, Lin et al. (2012) revealed new mechanisms by which curcumin inhibits the activation of HSCs in vitro. Curcumin dramatically attenuated AGEsmediated induction of RAGE gene expression, which is crucial for the activation of HSCs. Both PPAR $\gamma$ activity and de novo synthesis of glutathione (GSH) are required for the inhibitory effects of curcumin on $R A G E$ gene expression. The present findings showed that a PPAR $\gamma$ antagonist diminishes the beneficial effects of curcumin in HSCs whereas agonists have an effect similar to the natural compound. Furthermore, AGEs-induced production of reactive oxygen species and lipid peroxides was attenuated after curcumin exposure and accompanied by an increase in the activity of glutamatecysteine ligase (GCL) and the intracellular level of the reduced form of GSH. The inhibition of de novo synthesis of GSH using a GCL inhibitor partially reduced the effects of curcumin on RAGE gene expression. However, the suppression of either PPAR $\gamma$ or GCL did not completely eliminate the effects of curcumin as some inhibition of $R A G E$ expression on mRNA and protein levels was still detectable. This suggests the involvement of another mechanism(s), which requires further investigation. Although PPAR $\gamma$ acts as a transcription factor regulating gene expression upon binding to response elements in the promoters of target genes, Lin et al. did not find a consensus PPAR $\gamma$ binding site within RAGE gene promoter. Their preliminary results from promoter deletion assays indicate the presence of binding sites for other transcription factors, NF- $\mathrm{KB}$ and TCF/LEF1, where the latter mediates a nuclear response to WNT signals by interacting with $\beta$-catenin. Based on studies that showed the inhibition of NF- $\mathrm{BB}$ and disruption of WNT signalling after PPAR $\gamma$ activation in HSCs (Chen and Zheng, 2008), Lin et al. suggested that curcumin-induced increase in PPAR $\gamma$ negatively regulates

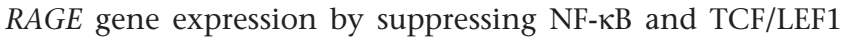
activities. Interestingly, PPARs have been demonstrated to be key regulators of glucose and lipid metabolism by regulating transcription of multiple target genes (Bermudez et al., 2010). $\operatorname{PPAR} \gamma$ agonists, thiazolidinediones, that increase tissue sensitivity to insulin and maintain plasma glucose level within normal range, are nowadays commonly used in the treatment of T2DM (Bermudez et al., 2010). However, these drugs produce adverse effects including serious heart failures. The opportunity for their replacement with naturally occurring compounds of low cytotoxicity such as curcumin would be an important milestone in the treatment of T2DM.

As shown in the previous studies, treatment of activated HSCs with curcumin increases PPAR $\gamma$ activity and PPAR $\gamma$ gene expression (Xu et al., 2003). Yet the mechanism of this action is unknown. The intriguing possibility may be linked to curcumin activity as an epigenetic agent involved in the regulation of activities of DNA methyltransferases (DNMTs). This polyphenol can covalently bind to the catalytic thiol group of Cys1226 present in DNMT1, which causes the inhibition of DNMT1 catalytic activity (Liu et al., 2009). This would explain the decrease in DNA promoter methylation upon curcumin exposure and reactivation of tumour suppressor genes, for example, RARbeta2 in human cervical cancer cells (Jha et al., 2010). PPAR $\gamma$ has been found to be silenced through its promoter methylation in colorectal cancer and this correlated with poor clinical outcome (Pancione et al., 2010). More notably, DNA hypermethylation of PPAR $\gamma$ promoter was observed in visceral adipose tissue of mouse models of diabetes and was associated with silencing of PPAR $\gamma$ expression that may contribute to the pathogenesis of diabetes (Fujiki et al., 2009).

Given that the present experiments were carried out in vitro in cultured HSCs, such studies in human patients are required to confirm the beneficial effect of curcumin in liver injuries developed in T2DM. Nevertheless, the study by Lin et al. strongly endorses the potential use of curcumin in T2DM therapy and opens up new avenues for therapeutic applications of this natural compound. It is conceivable that curcumin may confer hepatoprotection developed during the course of other chronic liver diseases. Importantly, the compound may ameliorate patient conditions in disorders where the lack of PPAR $\gamma$ activity contributes to disease pathogenesis such as obesity, atherosclerosis and multiple types of cancer.

\section{Conflict of interest}

The author has no conflict of interest to declare.

\section{References}

Aggarwal BB (2010). Targeting inflammation-induced obesity and metabolic diseases by curcumin and other nutraceuticals. Annu Rev Nutr 30: 173-199.

Basnet P, Skalko-Basnet N (2011). Curcumin: an anti-inflammatory molecule from a curry spice on the path to cancer treatment. Molecules 16: 4567-4598. 
Belkacemi A, Doggui S, Dao L, Ramassamy C (2011). Challenges associated with curcumin therapy in Alzheimer disease. Expert Rev Mol Med 13: e34.

Bermudez V, Finol F, Parra N, Parra M, Perez A, Penaranda L et al. (2010). PPAR-gamma agonists and their role in type 2 diabetes mellitus management. Am J Ther 17: 274-283.

Chen A, Zheng S (2008). Curcumin inhibits connective tissue growth factor gene expression in activated hepatic stellate cells in vitro by blocking NF-kappaB and ERK signalling. Br J Pharmacol 153: 557-567.

Fujiki K, Kano F, Shiota K, Murata M (2009). Expression of the peroxisome proliferator activated receptor gamma gene is repressed by DNA methylation in visceral adipose tissue of mouse models of diabetes. BMC Biol 7: 38.

Jha AK, Nikbakht M, Parashar G, Shrivastava A, Capalash N, Kaur J (2010). Reversal of hypermethylation and reactivation of the RARbeta2 gene by natural compounds in cervical cancer cell lines. Folia Biol (Praha) 56: 195-200.

Kang HC, Nan JX, Park PH, Kim JY, Lee SH, Woo SW et al. (2002). Curcumin inhibits collagen synthesis and hepatic stellate cell activation in-vivo and in-vitro. J Pharm Pharmacol 54: 119-126.

Kang Q, Chen A (2009). Curcumin suppresses expression of low-density lipoprotein (LDL) receptor, leading to the inhibition of LDL-induced activation of hepatic stellate cells. Br J Pharmacol 157: 1354-1367.

Lin J, Tang Y, Kang Q, Feng Y, Chen A (2012). Curcumin inhibits advanced glycation end-products (AGEs)-induced gene expression of receptor for AGEs (RAGE) in hepatic stellate cells in vitro by elevating PPAR $\gamma$ activity and attenuating oxidative stress. Br J Pharmacol 166: 2212-2227.

Liu Z, Xie Z, Jones W, Pavlovicz RE, Liu S, Yu J et al. (2009). Curcumin is a potent DNA hypomethylation agent. Bioorg Med Chem Lett 19: 706-709.
Pancione M, Sabatino L, Fucci A, Carafa V, Nebbioso A, Forte N et al. (2010). Epigenetic silencing of peroxisome proliferator-activated receptor gamma is a biomarker for colorectal cancer progression and adverse patients' outcome. PLoS ONE 5: e14229.

Ramasamy R, Yan SF, Schmidt AM (2009). RAGE: therapeutic target and biomarker of the inflammatory response - the evidence mounts. J Leukoc Biol 86: 505-512.

Rogers N, Stephenson M, Kitching A, Horowitz J, Coates P (2011). Amelioration of renal ischaemia-reperfusion injury by liposomal delivery of curcumin to renal tubular epithelial and antigen presenting cells. Br J Pharmacol 166: 194-209.

Rungseesantivanon S, Thenchaisri N, Ruangvejvorachai P, Patumraj S (2010). Curcumin supplementation could improve diabetes-induced endothelial dysfunction associated with decreased vascular superoxide production and PKC inhibition. BMC Complement Altern Med 10: 57.

Siu D (2010). A new way of targeting to treat coronary artery disease. J Cardiovasc Med (Hagerstown) 11: 1-6.

Tang Y, Chen A (2010). Curcumin prevents leptin raising glucose levels in hepatic stellate cells by blocking translocation of glucose transporter-4 and increasing glucokinase. Br J Pharmacol 161: 1137-1149.

World Health Organization, Media Centre (2011). Fact sheet No. 312, August 2011. Retrieved from http://www.who.int/mediacentre/ factsheets/fs312/en/index.html.

$\mathrm{Xu} \mathrm{J}, \mathrm{Fu} \mathrm{Y}$, Chen A (2003). Activation of peroxisome proliferator-activated receptor-gamma contributes to the inhibitory effects of curcumin on rat hepatic stellate cell growth. Am J Physiol Gastrointest Liver Physiol 285: G20-G30. 